\title{
Hyperglycemia as a Risk Factor for Atrial Fibrillation after Coronary Artery Bypass Graft Surgery
}

Y.E.Rezk ${ }^{1}$, E.S.Abdel-Azeem ${ }^{2}$, B.M.Aglan ${ }^{1}$ and M.A.Abd El Aty ${ }^{3}$

${ }^{1}$ Cardio thoracic Dept., Faculty of Medicine, Benha Univ., Benha, Egypt

${ }^{2}$ Anesthesia and Critical Care Dept., Faculty of Medicine, Benha Univ., Benha, Egypt

${ }^{3}$ Critical Care Medicine Dept., National Heart Institute, Cairo, Egypt

\begin{abstract}
Post-operative atrial fibrillation (POAF) is considered a common complication after coronary artery bypass surgery (CABG), which is associated with prolonged hospitalization, increased morbidity and even mortality [1]. We investigated the risk factors for POAF and analyzed the relationship between hyperglycemia and atrial fibrillation after CABG. This retrospective study was conducted on 100 patients who were admitted post CABG to critical care department at Benha University Hospital and National Heart Institute. Patients were divided into two groups: Group A included 50 patients who developed POAF and Group B included 50 patients who did not. In this study, we found that history of diabetes mellitus (DM), mean post-operative blood sugar (BS) and maximum post-operative BS were significant $(\mathrm{P}<0.05)$ in group A. We also found that post-operative drainage volume was significant in group A. We concluded that postoperative mean BS, maximum BS and post-operative drainage volume were significant risk factors for occurrence of POAF. We should control intensively blood sugar and also decreasing bleeding risks.
\end{abstract}

Keywords: CABG, POAF, Blood sugar, DM, Drainage volume.

\section{Introduction}

Atrial fibrillation $(\mathrm{AF})$ is the most common cardiac arrhythmia in adults and is characterized by chaotic atrial electrical activity leading to irregular heart rhythm [1].

Postoperative atrial fibrillation (POAF) is defined as new-onset atrial fibrillation $(\mathrm{AF})$ in the initial period after surgery and is considered the most important type of secondary $\mathrm{AF}$ ( $\mathrm{AF}$ resulting from an identifiable, primary, acute condition) [2].

POAF is a complication in aPP.roximately one-third of patients undergoing cardiac surgery, causing substantial increases in hospital costs; intensive care unit time and hospital stay [3].

Diabetes mellitus is a group of metabolic disorders where there are high blood sugar levels over time. Prolonged elevations in sugar levels lead to a number of health complications including cardiovascular disease and kidney disease $[4,5]$.

In a comprehensive meta-analysis, diabetic patients were found to have an $40 \%$ greater risk for developing atrial fibrillation compared to non-diabetic patients [5] and a more recent meta-analysis identified a $20 \%$ increase in the risk of developing atrial fibrillation for prediabetic patients whereas in patients with diabetes, this number was elevated to $28 \%$ greater change of atrial fibrillation development [6].

$\mathrm{AF}$ is clinically managed through pharmacologic heart rate control with beta-blockers, calcium-channel blockers, and other antiarrhythmic medications [7]. A rhythm control strategy can be attempted to return the patient to sinus rhythm via pharmacologic or electrical cardioversion, or increasingly by intracardiac catheter ablation [8].

Intensive care unit (ICU) protocols currently use bedside glucometers to check glucose levels every
30-60 minutes in the immediate post-operative period and adjust insulin titrations accordingly [9].

The aims of the present study were to identify predictors of postoperative $\mathrm{AF}$ after $\mathrm{CABG}$, and to show the relationship between postoperative blood sugar concentration (BS) and postoperative AF.

\section{Patients and methods}

A 100 Patients post CABG admitted to critical care department at Benha University Hospital were divided into two groups:

* Group [A] included 50 patients: Patients who developed POAF.

* Group [B] included 50 patients: Patients who did not develop POAF.

Patients were monitored postoperatively and arrhythmias were recorded using a 12 leads ECG and blood sugar levels were also recorded every 2 hours in the ICU in the first 6 hours after operation and subsequently every 6 hours. Other post-operative routine labs were also recorded. Both groups were compared according to the preoperative and postoperative data.

\section{Results}

The collected data were tabulated and statistically analyzed using SPSS program software version 18.0. Quantitative data presented as minimum and maximum of the range and the mean \pm SD while Qualitative data presented as number and percentage. $P$ values less than 0.05 were considered significant.

There were no significant differences ( $\mathrm{p}$-value $>0.05$ ) between both groups as regard age ,sex ,preoperative heart rate and blood pressure (systolic and diastolic) ,medical history as regard to smoking and hypertension but history of DM was significant in group A (P-value< 0.001$)$ as shown in Table (1) 
Table (1) Comparison between the two groups as regard age, sex, risk factors [Medical history] and vital data.

\begin{tabular}{|c|c|c|c|c|}
\hline & \multirow{2}{*}{$\begin{array}{c}\text { Group A } \\
(n=50)\end{array}$} & \multirow{2}{*}{$\begin{array}{c}\text { Group B } \\
(\mathbf{n}=\mathbf{5 0})\end{array}$} & \multicolumn{2}{|c|}{ Tests } \\
\hline & & & $T$ or $X^{2}$ & P-value \\
\hline \multicolumn{5}{|c|}{ Age } \\
\hline Range & $31-70$ & $44-69$ & 0.599 & 0.551 \\
\hline Mean \pm SD & $58.36 \pm 10.33$ & $59.44 \pm 7.47$ & & \\
\hline \multicolumn{5}{|c|}{ Sex } \\
\hline Female & $8(16 \%)$ & $12(24 \%)$ & 1.000 & 0.317 \\
\hline Male & $42(84 \%)$ & $38(76 \%)$ & & \\
\hline \multicolumn{5}{|l|}{ Medical history } \\
\hline Smoking & $41(82 \%)$ & $45(90 \%)$ & 1.329 & 0.249 \\
\hline Diabetes Mellitus & $36(72 \%)$ & $15(30 \%)$ & 17.647 & $<0.001 * *$ \\
\hline Hypertension & $27(54 \%)$ & $20(40 \%)$ & 1.967 & 0.161 \\
\hline \multicolumn{5}{|c|}{ Preoperative Heart rate (beat/min) } \\
\hline Mean \pm SD & $85.60 \pm 9.62$ & $84.48 \pm 8.99$ & 0.602 & 0.549 \\
\hline \multicolumn{5}{|c|}{ Diastolic Blood pressure $(\mathrm{mm} / \mathbf{H g})$} \\
\hline Mean \pm SD & $86.60 \pm 14.23$ & $87.20 \pm 14.00$ & 0.213 & 0.832 \\
\hline \multicolumn{5}{|c|}{ Systolic Blood pressure (mm/Hg) } \\
\hline Mean \pm SD & $131.80 \pm 20.77$ & $133.40 \pm 20.06$ & 0.392 & 0.696 \\
\hline
\end{tabular}

There was no significant difference ( $\mathrm{p}$-value $>0.05$ ) in both groups according to pre-operative echo findings and postoperative ejection fraction as shown in Table (2).

Table (2) Comparison between the two groups as regard Preoperative Echo Findings and Post-operative ejection fraction $(\mathrm{EF})$.

\begin{tabular}{|c|c|c|c|c|c|c|}
\hline & \multicolumn{2}{|c|}{$\begin{array}{c}\text { Group A } \\
(\mathbf{n}=\mathbf{5 0})\end{array}$} & \multicolumn{2}{|c|}{$\begin{array}{c}\text { Group B } \\
(\mathrm{n}=50)\end{array}$} & \multicolumn{2}{|c|}{ Chi- square } \\
\hline & $\mathbf{N}$ & $\%$ & $\mathbf{N}$ & $\%$ & $\mathbf{X} 2$ & P-value \\
\hline Preoperative Echo Findings & & & & & & \\
\hline Normal & 18 & 36 & 23 & 46 & 2.047 & 0.563 \\
\hline Dilated left atrium( LA) & 15 & 30 & 11 & 22 & & \\
\hline $\begin{array}{l}\text { Dilated left ventricle( LV) - dilated } \\
\text { LA - low EF }\end{array}$ & 9 & 18 & 6 & 12 & & \\
\hline $\begin{array}{l}\text { Regional wall Motion Abnormality } \\
\text { Post-Operative Ejection Fraction (EF) }\end{array}$ & 8 & 16 & 10 & 20 & & \\
\hline Normal $(E F>55 \%)$ & 26 & 52 & 22 & 44 & 0.707 & 0.702 \\
\hline Borderline (EF35-55) & 12 & 24 & 15 & 30 & & \\
\hline Impaired $(\mathbf{E F}<35)$ & 12 & 24 & 13 & 26 & & \\
\hline
\end{tabular}

There was no statistically significant difference $(\mathrm{P}>0.05)$ between the two groups as regard preoperative Fasting Blood Sugar level. Post-operative mean and maximum blood sugar level showed statistically significance $(\mathrm{P}-\mathrm{Value}<$ 0.05 ) between the two groups which was significant in group A as shown in Table (3).

Table (3) Comparison between the two groups as regard preoperative Fasting Blood Sugar level, Post-operative Mean and Maximum blood sugar level.

\begin{tabular}{|c|c|c|c|c|c|c|c|c|}
\hline \multirow[t]{2}{*}{ Blood Sugar Level(mg/dl) } & \multicolumn{3}{|c|}{$\begin{array}{c}\text { Group A } \\
(\mathbf{n}=\mathbf{5 0})\end{array}$} & \multicolumn{3}{|c|}{$\begin{array}{c}\text { Group B } \\
(n=50)\end{array}$} & \multicolumn{2}{|c|}{ T-test } \\
\hline & Mean & \pm & SD & Mean & \pm & SD & $\mathbf{T}$ & P-value \\
\hline $\begin{array}{l}\text { Preoperative Fasting blood } \\
\text { sugar }\end{array}$ & 115.12 & \pm & 18.8 & 122.16 & \pm & 20.49 & 1.790 & 0.077 \\
\hline $\begin{array}{l}\text { Postoperative Mean blood } \\
\text { sugar }\end{array}$ & 176.68 & \pm & 53.65 & 157.88 & \pm & 27.81 & 2.200 & $0.030 *$ \\
\hline $\begin{array}{l}\text { Postoperative Maximum } \\
\text { blood sugar }\end{array}$ & 277.37 & \pm & 58.91 & 255.00 & \pm & 47.01 & 2.098 & $0.038 *$ \\
\hline
\end{tabular}


There was no statistically significant difference (Pvalue $>0.05$ ) between the two groups as regard Bypass Time, Cross Clamping Time, Ventilation Time, ICU stay time, Blood and Blood products transfusion, postoperative Volume Balance, presence of intra- aortic balloon. There was statistically significant difference (P-value < 0.05) between the two groups as regard postoperative drainage volume that was significant in group A than group B as shown in Table (4).

Table (4) Comparison between the two groups as regard Surgery steps and Post-operative period characters and Events.

\begin{tabular}{|c|c|c|c|c|}
\hline & \multirow{2}{*}{$\begin{array}{c}\text { Group A } \\
(n=50)\end{array}$} & \multirow{2}{*}{$\begin{array}{c}\text { Group B } \\
(\mathbf{n}=\mathbf{5 0})\end{array}$} & \multicolumn{2}{|c|}{ Tests } \\
\hline & & & T or $X^{2}$ & P-value \\
\hline \multicolumn{5}{|c|}{ Bypass Time (min.) } \\
\hline Range & $60-140$ & $60-140$ & \multirow{2}{*}{0.117} & \multirow{2}{*}{0.907} \\
\hline Mean \pm SD & $90.00 \pm 25.39$ & $89.40 \pm 25.75$ & & \\
\hline \multicolumn{5}{|c|}{ Cross Clamping Time /min } \\
\hline Range & $30-110$ & $30-105$ & \multirow{2}{*}{1.318} & \multirow{2}{*}{0.191} \\
\hline Mean \pm SD & $48.10 \pm 24.01$ & $54.40 \pm 23.79$ & & \\
\hline \multicolumn{5}{|c|}{ Ventilation Time/ hour } \\
\hline Range & $6-35$ & $6-47$ & \multirow{2}{*}{0.926} & \multirow{2}{*}{0.357} \\
\hline Mean \pm SD & $12.00 \pm 6.66$ & $13.56 \pm 9.88$ & & \\
\hline \multicolumn{5}{|c|}{ ICU stay time /day } \\
\hline Range & $2-6$ & $2-6$ & \multirow{2}{*}{1.523} & \multirow{2}{*}{0.131} \\
\hline Mean \pm SD & $3.1 \pm 1.45$ & $2.72 \pm 1.01$ & & \\
\hline \multicolumn{5}{|c|}{ Blood and Blood products transfusion } \\
\hline Fresh Blood & $32(64 \%)$ & $36(72 \%)$ & 0.735 & 0.391 \\
\hline Packed RBCS & $36(72 \%)$ & $40(80 \%)$ & 0.877 & 0.349 \\
\hline PLASMA & $37(74 \%)$ & $41(82 \%)$ & 0.932 & 0.334 \\
\hline \multicolumn{5}{|c|}{ Postoperative Volume Balance } \\
\hline Positive & $5(10 \%)$ & $4(8 \%)$ & \multirow{2}{*}{0.122} & \multirow{2}{*}{0.727} \\
\hline Negative & $45(90 \%)$ & $46(92 \%)$ & & \\
\hline \multicolumn{5}{|c|}{ Postoperative drainage volume (cc) } \\
\hline Range & $500-1800$ & $400-1200$ & \multirow{2}{*}{6.118} & \multirow{2}{*}{$<0.001 * *$} \\
\hline Mean \pm SD & $1164.00 \pm 401.91$ & $768.00 \pm 218.94$ & & \\
\hline \multicolumn{5}{|c|}{ Intraortic balloon } \\
\hline Yes & $9(18 \%)$ & $5(10 \%)$ & \multirow{2}{*}{1.329} & \multirow{2}{*}{0.249} \\
\hline No & $41(82 \%)$ & $45(90 \%)$ & & \\
\hline
\end{tabular}

\section{Discussion}

In our study, There were no significant differences ( $p$-value $>0.05$ ) between both groups as regard age, sex ,pre-operative heart rate and blood pressure (systolic and diastolic), medical history as regard to smoking and hypertension but history of DM was significant in group A (P-value< 0.001). According to Tatsuishi et al, 2017 age was identified as an independent risk factor for POAF that could be attributed to Age-related comorbidities [10, 11]

Amar et al, 2002, concluded that aging causes some changes as "shorter effective refractoriness, delayed SA and AV nodal conduction times, atrial stiffening, and splitting of the atrial excitation waveform caused by the pectinated trabeculae" [12].

In our study history of DM showed statistically significance between the two groups with (P-value $<0.001)$. That also was significant according to studies of Tatsuishi et al, 2017 but did not show significance according to $[10,11,13]$
These findings were suPP.orted by Benjamin et al, 1994, who reported that DM is established as an independent risk factor for atrial fibrillation (AF) after 38 years of follow-up [14].

In our study, postoperative Mean blood sugar showed statistically significance (P-value 0.030) between the two groups which also was statistically significance according to Tatsuishi et al, 2017 and in our study Maximum blood sugar also showed statistically significance between the two groups with( p-value of 0.038).

According to Ismail et al, 2017 ventilation time and ICU stay time showed significance (p-value < 0.05 ) between the two groups which was explained as a result of arrhythmia but in our study and according to Tatsuishi et al, 2017 no significance detected as regard ventilation time and ICU stay ( $\mathrm{p}$-value $>0.05$ ).

According to Ismail et al, 2017 preoperative heart rate, LA volume and depressed preoperative EF, showed significant $(\mathrm{p}$-value $<0.05)$ between the two 
groups .Enlarged LA size is consistently reproducible predictors for POAF. The influence of enlarged left atrial on POAF has been demonstrated with $\mathrm{CABG}$ in several studies [15].

\section{Conclusion}

We concluded that post-operative mean BS, maximum BS and post-operative drainage volume were significant risk factors for occurrence of POAF. In postoperative period, blood sugar should be tightly controlled and also preventing and managing any post-operative bleeding to make post-operative drainage volume as less as possible and making patient hemodynamic status more stable.

\section{References}

[1] E. J.Benjamin, S. S.Virani, C. W.Callaway, A. M.Chamberlain, A. R.Chang, S.Cheng, R.Deo, Forecasting the future of cardiovascular disease in the United States: a policy statement from the American Heart Association. Circulation.,,Vol. 137(12), PP.e67-e492, 2018.

[2] S. A.Lubitz, X.Yin, M.Rienstra, R. B.Schnabel, A. J.Walkey, J. W.Magnani, D.Levy, Long-term outcomes of secondary atrial fibrillation in the community: the Framingham Heart Study. Circulation, Vol. 131(19), PP.1648-1655,2015.

[3] J. W.Greenberg, T. S.Lancaster, R. B.Schuessler, S. J.Melby, Postoperative atrial fibrillation following cardiac surgery: a persistent complication. European Journal of CardioThoracic Surgery, Vol. 52(4), PP.665-672,2017.

[4] R. R.Huxley, K. B.Filion, S.Konety, A.Alonso, Meta-analysis of cohort and case-control studies of type 2 diabetes mellitus and risk of atrial fibrillation. The American journal of cardiology, Vol.108(1), PP.56-62,2011.

[5] J. M.Forbes, M. E.Cooper, Mechanisms of diabetic complications. Physiological reviews., Vol.93(1), PP.137-188,2013.

[6] D.Aune, T.Feng, S.Schlesinger, I.Janszky, T.Norat, E.Riboli, Diabetes mellitus, blood glucose and the risk of atrial fibrillation: A systematic review and meta-analysis of cohort studies. Journal of Diabetes and its Complications, Vol.32(5), PP.501-511,2018.
[7] I. C.VanGelder, M.Rienstra, H. J.Crijns, B.Olshansky, Rate control in atrial fibrillation. The Lancet, Vol.388(10046), PP.818-828,2016.

[8] J. P.Piccini, L.Fauchier, Rhythm control in atrial fibrillation. The Lancet, Vol.388(10046), PP.829840,2016.

[9] A. P.Furnary, K. J.Zerr, G. L.Grunkemeier, A.Starr, Continuous intravenous insulin infusion reduces the incidence of deep sternal wound infection in diabetic patients after cardiac surgical procedures. The Annals of thoracic surgery, Vol.67(2), PP.352-360,1999.

[10]W.Tatsuishi, H.Adachi, M.Murata, J.Tomono, S.Okonogi, S.Okada, S.Ohshima, Postoperative hyperglycemia and atrial fibrillation after coronary artery bypass graft surgery. Circulation Journal, Vol. 79(1), PP.112-118,2014.

[11] M. F.Ismail, A. F.El-Mahrouk, T. H.Hamouda, H.Radwan, A.Haneef, A. A.Jamjoom, Factors influencing postoperative atrial fibrillation in patients undergoing on-pump coronary artery bypass grafting, single center experience. Journal of cardiothoracic surgery, Vol.12(1), PP.40,2017.

[12]D.Amar, H.Zhang, D. H.Leung, N.Roistacher, A. H.Kadish, Older age is the strongest predictor of postoperative atrial fibrillation. Anesthesiology: The Journal of the American Society of Anesthesiologists, Vol.96(2), PP.352-356,2002.

[13]S.Omer, L. D.Cornwell, A.Bakshi, E.Rachlin, O.Preventza, T. K.Rosengart, G. Pattakos, Incidence, predictors, and impact of postoperative atrial fibrillation after coronary artery bypass grafting in military veterans. Texas Heart Institute Journal, Vol.43(5), PP.397403,2016.

[14] E. J.Benjamin, D.Levy, S. M.Vaziri, R. B.D'Agostino, A. J.Belanger, P. A.Wolf, Independent risk factors for atrial fibrillation in a population-based cohort: the Framingham Heart Study. Jama, Vol.271(11), PP.840-844,1994.

[15] M.Osranek, K.Fatema, F.Qaddoura, A.AlSaileek, M. E.Barnes, K. R.Bailey, J. B. Seward, Left atrial volume predicts the risk of atrial fibrillation after cardiac surgery: a prospective study. Journal of the American College of Cardiology, Vol. 48(4), PP.779-786,2006. 